\title{
Monodromy and K-theory of Schubert curves via generalized jeu de taquin
}

\author{
Maria Monks Gillespie $\rrbracket^{\Perp}$ and Jake Levinson非 \\ ${ }^{1}$ University of California, Berkeley, CA. \\ ${ }^{2}$ University of Michigan, Ann Arbor, MI.
}

\begin{abstract}
We establish a combinatorial connection between the real geometry and the $K$-theory of complex Schubert curves $\mathcal{S}\left(\lambda_{\bullet}\right)$, which are one-dimensional Schubert problems defined with respect to flags osculating the rational normal curve. In a previous paper, the second author showed that the real geometry of these curves is described by the orbits of a map $\omega$ on skew tableaux, defined as the commutator of jeu de taquin rectification and promotion. In particular, the real locus of the Schubert curve is naturally a covering space of $\mathbb{R P}^{1}$, with $\omega$ as the monodromy operator.

We provide a fast, local algorithm for computing $\omega$ without rectifying the skew tableau, and show that certain steps in our algorithm are in bijective correspondence with Pechenik and Yong's genomic tableaux, which enumerate the $K$-theoretic Littlewood-Richardson coefficient associated to the Schubert curve. Using this bijection, we give purely combinatorial proofs of several numerical results involving the $K$-theory and real geometry of $\mathcal{S}\left(\lambda_{\mathbf{0}}\right)$.

Résumé. Nous établissons une connection entre la géométrie réelle et la K-théorie des courbes de Schubert $\mathcal{S}\left(\lambda_{\bullet}\right)$. Ces dernières sont des problèmes de Schubert, de dimension 1, définies par rapport à des drapeaux tangents à la courbe rationelle normale. Le deuxième auteur a démontré auparavant que la géométrie de ces courbes est décrite par les orbites d'une transformation $\omega$ de tableaux de Young gauches : le commutateur de la rectification (au sens du jeu de taquin de Schützenberger) et de la promotion. Les points réels de $\mathcal{S}\left(\lambda_{\bullet}\right)$ forment alors un revêtement de $\mathbb{R P}^{1}$ avec $\omega$ comme opérateur de monodromie.

Nous introduisons un algorithme local et rapide qui permet de calculer $\omega$ sans devoir rectifier le tableau. Nous démontrons ensuite que certaines étapes de l'algorithme sont en bijection avec les tableaux génomiques de PechenikYong, lesquels énumèrent le coefficient de Littlewood-Richardson K-théorique associé à $\mathcal{S}\left(\lambda_{\bullet}\right)$. Finalement, nous démontrons de façon purement combinatoire certaines propriétés géométriques et K-théoriques de $\mathcal{S}\left(\lambda_{\bullet}\right)$.
\end{abstract}

Keywords. Young tableaux, monodromy, Schubert calculus, $K$-theory, osculating flag, jeu de taquin

\section{Introduction}

This paper is an abridged version of the full paper [5] by the authors. We study the real and complex geometry of certain one-dimensional intersections $S$ of Schubert varieties defined with respect to 'osculating' flags. These curves are known to have smooth real points, which naturally cover the circle $\mathbb{R P}^{1}$;

${ }^{\dagger}$ Email: monks@math. berkeley.edu. Supported by NSF GRFP and Hertz Foundation.

‡Email: jakelev@umich. edu. Supported by FRQNT B2-184146 and by NSF grants DMS-1160720, 1464693 and 1101152. 
we give a new combinatorial rule, in terms of certain Young tableaux, for the monodromy operator on the fibers (a more complicated rule was given in [9]). Our rule is fast and combinatorially 'local', making it easier to count $\eta(S)$, the number of components of $S(\mathbb{R})$, which fully characterizes the real topology. Moreover, our rule computes the class of $S$ in the $K$-theory of the Grassmannian: it explicitly produces Pechenik and Yong's genomic tableaux [12]. This connection gives rise to purely combinatorial proofs of two known geometric relations between $\eta(S)$ and the Euler characteristic $\chi\left(\mathcal{O}_{S}\right)$, and also yields new facts about the real and complex geometry of $S$.

To define the curve $S$, recall first that the rational normal curve is the image of the embedding $\mathbb{P}^{1} \hookrightarrow$ $\mathbb{P}^{n-1}=\mathbb{P}\left(\mathbb{C}^{n}\right)$ by the Veronese map

$$
t \mapsto\left[1: t: t^{2}: \cdots: t^{n-1}\right] .
$$

Let $\mathcal{F}_{t}$ be the osculating or maximally tangent flag to this curve at $t \in \mathbb{P}^{1}$, i.e. the complete flag in $\mathbb{C}^{n}$ formed by the iterated derivatives of this map. Let $G\left(k, \mathbb{C}^{n}\right)$ be the Grassmannian, and $\Omega\left(\lambda, \mathcal{F}_{t}\right)$ the Schubert variety for the condition $\lambda$ with respect to $\mathcal{F}_{t}$. The Schubert curve is the intersection

$$
S=S\left(\lambda_{1}, \ldots, \lambda_{r}\right)=\Omega\left(\lambda_{1}, \mathcal{F}_{t_{1}}\right) \cap \cdots \cap \Omega\left(\lambda_{r}, \mathcal{F}_{t_{r}}\right),
$$

where the osculation points $t_{i}$ are real numbers with $0=t_{1}<t_{2}<\cdots<t_{r}=\infty$, and $\lambda_{1}, \ldots, \lambda_{r}$ are partitions for which $\sum\left|\lambda_{i}\right|=k(n-k)-1$. For simplicity, we always consider intersections of only three Schubert varieties, though the results of this paper (in particular, Theorems 1.2, 1.5 and 1.6 extend to the general case without difficulty. With this in mind, we let $\alpha, \beta, \gamma$ be partitions with $|\alpha|+|\beta|+|\gamma|=$ $k(n-k)-1$, and we consider the Schubert curve

$$
S(\alpha, \beta, \gamma)=\Omega\left(\alpha, \mathcal{F}_{0}\right) \cap \Omega\left(\beta, \mathcal{F}_{1}\right) \cap \Omega\left(\gamma, \mathcal{F}_{\infty}\right) .
$$

Schubert varieties with respect to such osculating flags have been studied extensively in the context of degenerations of curves [2] [3] [11], Schubert calculus and the Shapiro-Shapiro Conjecture [10] [14] [16], and the geometry of the moduli space $\overline{M_{0, r}}(\mathbb{R})$ [17]. They satisfy unusually strong transversality properties, particularly when the osculation points $t$ are chosen to be real [3] [10]; in particular, $S$ is known to be one-dimensional (if nonempty) and reduced [9]. Moreover, intersections of such Schubert varieties in dimensions zero and one have been found to have remarkable topological descriptions in terms of Young tableau combinatorics. [2] [9] [13] [17]

The Schubert curve is no exception: recent work ([9]) has shown that its real connected components can be described by combinatorial operations, related to jeu de taquin and Schützenberger's promotion and evacuation, on chains of skew Young tableaux. Recall that a skew semistandard Young tableau is Littlewood-Richardson if its reading word is ballot, meaning that every suffix of the reading word has partition content.

Definition 1.1 We write $\operatorname{LR}\left(\lambda_{1}, \ldots, \lambda_{r}\right)$ to denote the set of sequences $\left(T_{1}, \ldots, T_{r}\right)$ of skew LittlewoodRichardson tableaux, filling a $k \times(n-k)$ rectangle, such that the shape of $T_{i}$ extends that of $T_{i-1}$ and $T_{i}$ has content $\lambda_{i}$ for all $i$. (The tableaux $T_{1}$ and $T_{r}$ are uniquely determined and may be omitted.)

The theorem below describes the topology of $S(\alpha, \beta, \gamma)(\mathbb{R})$ in terms of tableaux:

Theorem 1.2 ([9], Corollary 4.9) There is a map $S \rightarrow \mathbb{P}^{1}$ that makes the real locus $S(\mathbb{R})$ a smooth covering of the circle $\mathbb{R P}^{1}$. The fibers over 0 and $\infty$ are in canonical bijection with, respectively, 
$\operatorname{LR}(\alpha, \square, \beta, \gamma)$ and $\operatorname{LR}(\alpha, \beta, \square, \gamma)$. Under this identification, the arcs of $S(\mathbb{R})$ covering $\mathbb{R}_{-}$induce the jeu de taquin bijection

$$
\operatorname{sh}: \operatorname{LR}(\alpha, \beta, \square, \gamma) \rightarrow \operatorname{LR}(\alpha, \square, \beta, \gamma),
$$

and the arcs covering $\mathbb{R}_{+}$induce a different bijection esh, called evacuation-shuffling. The monodromy operator $\omega$ is, therefore, given by $\omega=$ sh $\circ$ esh.

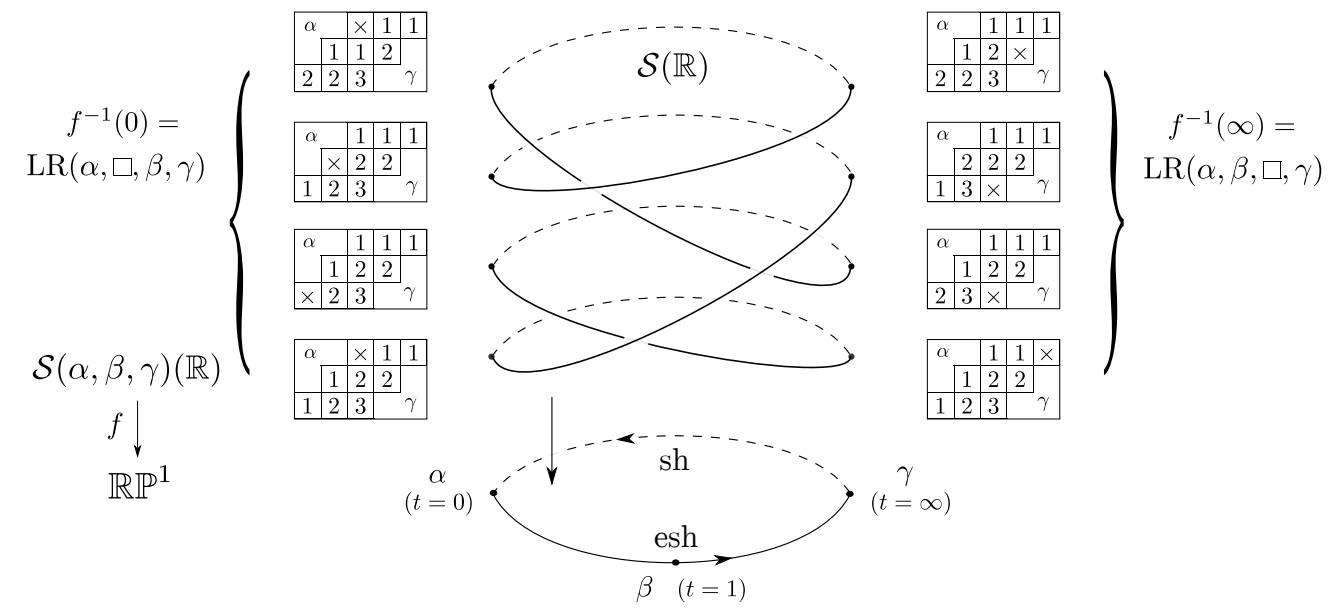

Figure 1: An example of the covering space of Theorem 1.2. The fibers over 0 and $\infty$ are indexed by chains of tableaux, with $\otimes$ denoting the single box. The dashed arcs correspond to sliding the $\bowtie$ through the tableau using jeu de taquin. The monodromy operator is $\omega=$ sh $\circ$ esh.

The operators esh and $\omega$ are our objects of study. In [9], the second author described esh as the conjugation of jeu de taquin promotion by rectification (see Section 2 for a precise definition). Variants of this operation have appeared elsewhere in [1], [7], [8].

We prove two main theorems. The first is a shorter, 'local' combinatorial description of the map esh, which no longer requires rectifying or otherwise modifying the skew shape. We call our algorithm local evacuation shuffling. Local evacuation-shuffling resembles jeu de taquin: it consists of successively moving the $\bowtie$ through $T$ through a weakly increasing sequence of squares. Unlike JDT, the path is in general disconnected. (See Section 3 for the definition, and Figure 2 for a visual description of the path of the $\bigotimes$.)

Theorem 1.3 The map esh agrees with local evacuation shuffling. In particular, $\omega=$ sh $\circ$ local-esh.

Our second main result is related to K-theory $K\left(G\left(k, \mathbb{C}^{n}\right)\right)$ and the orbit structure of $\omega$. We first recall a key consequence of Theorem 1.2 .

Proposition 1.4 ([9], Lemma 5.6) Let $S$ have $\iota(S)$ irreducible components and let $S(\mathbb{R})$ have $\eta(S)$ connected components. Let $\chi\left(\mathcal{O}_{S}\right)$ be the holomorphic Euler characteristic. Then

$$
\begin{aligned}
& \eta(S) \geqslant \iota(S) \geqslant \chi\left(\mathcal{O}_{S}\right) \text { and } \\
& \eta(S) \equiv \chi\left(O_{S}\right) \quad(\bmod 2)
\end{aligned}
$$




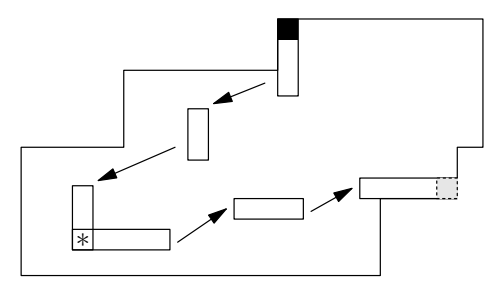

Figure 2: The path of the $₫$ in a local evacuation-shuffle. The black and gray squares are the initial and final locations of the $\otimes$; the algorithm switched from "Phase 1" to "Phase 2 " at the square marked by a $*$. There is an antidiagonal symmetry: the Phase 1 path forms a vertical strip, while the Phase 2 path forms a horizontal strip. We give a precise statement of this symmetry in the full paper.

We note that $\eta(S)$ is the number of orbits of $\omega$, viewed as a permutation of $\operatorname{LR}(\alpha, \square, \beta, \gamma)$. The statements above are most interesting in the context of K-theoretic Schubert calculus, which expresses $\chi\left(\mathcal{O}_{S}\right)$ in terms of both of ordinary and (K-theoretic) genomic Young tableaux, namely

$$
\chi\left(\mathcal{O}_{S}\right)=|\operatorname{LR}(\alpha, \square, \beta, \gamma)|-\left|K\left(\gamma^{c} / \alpha ; \beta\right)\right| .
$$

See Section 4 for the definition of $K\left(\gamma^{c} / \alpha ; \beta\right)$, due to Pechenik-Yong [12]. In particular, we see that

$$
\begin{aligned}
& \left|K\left(\gamma^{c} / \alpha ; \beta\right)\right| \geqslant|\operatorname{LR}(\alpha, \beta, \square, \gamma)|-|\operatorname{orbits}(\omega)|, \text { and } \\
& \left|K\left(\gamma^{c} / \alpha ; \beta\right)\right| \equiv|\operatorname{LR}(\alpha, \beta, \square, \gamma)|-|\operatorname{orbits}(\omega)| \quad(\bmod 2) .
\end{aligned}
$$

We reformulate as follows: recall that the reflection length of a permutation $\sigma \in S_{N}$ is the minimum length of a factorization of $\sigma$ into arbitrary (not necessarily adjacent) transpositions. We have

$$
\operatorname{rlength}(\sigma)=\sum_{\mathcal{O} \in \operatorname{orbits}(\sigma)}(|\mathcal{O}|-1)=N-|\operatorname{orbits}(\sigma)| \text {. }
$$

We also recall that the sign of a permutation is the parity of the reflection length,

$$
\operatorname{sgn}(\sigma) \equiv \operatorname{rlength}(\sigma) \quad(\bmod 2),
$$

where we use the convention that the sign of a permutation is 0 or 1 (rather than \pm 1 ). Thus,

$$
\begin{aligned}
& \left|K\left(\gamma^{c} / \alpha ; \beta\right)\right| \geqslant \operatorname{rlength}(\omega), \text { and } \\
& \left|K\left(\gamma^{c} / \alpha ; \beta\right)\right| \equiv \operatorname{sgn}(\omega) \quad(\bmod 2) .
\end{aligned}
$$

For the case where $\beta$ is a horizontal strip, a combinatorial interpretation of these facts was given in [9], indexing all but one step of an orbit by genomic tableaux. Our second main result generalizes this combinatorial interpretation, showing that certain steps of local evacuation-shuffling correspond bijectively to the genomic tableaux $K\left(\gamma^{c} / \alpha ; \beta\right)$ :

Theorem 1.5 As $T$ ranges over $\operatorname{LR}(\alpha, \square, \beta, \gamma)$, for either phase of the local description of $\operatorname{esh}(T)$, the gaps in the $₫$ path are in bijection with the set $K\left(\gamma^{c} / \alpha ; \beta\right)$. 
Using the bijections of Theorem 1.5, we give an independent, purely combinatorial proof of the relations (3) and (4), by factoring $\omega$ into auxiliary operators $\omega_{i}$, which roughly correspond to the individual steps of local evacuation-shuffling, applied in isolation. If $\beta$ has $\ell(\beta)$ parts, we have the following:

Theorem 1.6 There is a factorization of $\omega$ as a composition $\omega_{\ell(\beta)} \cdots \omega_{1}$, such that for every $i$, and every orbit $\mathcal{O}$ of $\omega_{i}$, the bijections of Theorem 1.5 yield exactly $|\mathcal{O}|-1$ distinct genomic tableaux.

By summing over the orbits of the $\omega_{i}$ 's, we deduce

$$
\operatorname{rlength}(\omega) \leqslant \sum_{i} \operatorname{rlength}\left(\omega_{i}\right)=\sum_{i, \mathcal{O}}(|\mathcal{O}|-1)=\left|K\left(\gamma^{c} / \alpha ; \beta\right)\right|,
$$

by the subadditivity of reflection length. The sign computation is analogous.

The remainder of this paper is structured as follows. In Section 3, we define local-esh and sketch the proof that it agrees with esh. Section 4 contains the link to K-theory and proof sketches of Theorems 1.5 and 1.6 Sections 5 and 6 explore some consequences of the main results.

\section{Background}

\subsection{Tableaux and shuffling}

We refer to [4] for the standard definitions of partitions, semistandard Young tableaux and jeu de taquin. We briefly state some additional conventions that we will use.

Let $\lambda=\left(\lambda_{1} \geqslant \cdots \geqslant \lambda_{k}\right)$ be a partition. We will refer to the partition $\lambda$ and its Young diagram interchangeably throughout, using the English convention for Young diagrams. If $\mu$ is a partition with $\mu_{i} \leqslant \lambda_{i}$ for all $i$, then the skew shape $\lambda / \mu$ is the diagram formed by deleting the squares of $\mu$ from that of $\lambda$. Its size, written $|\lambda / \mu|$, is the number of squares that remain in the diagram. We will occasionally refer to (co-)corners of a skew shape. The inner (respectively, outer) corners of $\lambda / \mu$ are the corners of $\lambda$ (respectively, the co-corners of $\mu$ ). These are the squares which, if deleted, leave a smaller skew shape. Similarly, the inner (resp. outer) co-corners are the co-corners of $\lambda$ (resp. the corners of $\mu$ ): the exterior squares which can be added to obtain a larger skew shape.

We write $\boxplus=\left((n-k)^{k}\right)$ to denote a fixed rectangular shape of size $k \times(n-k)$, and we will always work with skew shapes that fit inside $\boxplus$. The complementary partition to $\lambda \subset \boxplus$, denoted $\lambda^{c}$, is the partition $\left(n-k-\lambda_{k}, n-k-\lambda_{k-1}, \ldots, n-k-\lambda_{1}\right)$.

Let $T$ be a semistandard Young tableau of shape $\lambda / \mu$. The reading word of $T$ is the sequence formed by reading the rows from bottom to top, and left to right within a row. The suffix of an entry $m$ of $T$ is the suffix of the reading word consisting of the letters strictly after $m$. The weak suffix is the suffix including that letter and those after it. A suffix is ballot for $(i, i+1)$ if it contains at least as many $i$ 's as $i+1$ 's, and is tied if it has the same number of $i$ 's as $i+1$ 's. Finally, $T$ is ballot or Littlewood-Richardson (also known as Yamanouchi or lattice) if every weak suffix of its reading word is ballot for $(i, i+1)$, for all $i$.

Let $S, T$ be semistandard skew tableaux, such that the shape of $T$ extends the shape of $S$, that is, $T$ can be formed by successively adding outer co-corners starting from $S$. Let $S^{\prime}$ and $T^{\prime}$ respectively be the tableaux formed by performing successive outward (resp. inward) jeu de taquin slides on $S$ (resp. T), using the entries of $T$ in ascending (resp. $S$, descending) order, and ordering equal entries of $T$ from left to right (resp. $S$, right to left).

Definition 2.1 The (jeu de taquin) shuffle of $(S, T)$, denoted $\operatorname{sh}(S, T)$, is the pair of tableaux $\left(T^{\prime}, S^{\prime}\right)$. 
The rectification of a skew tableau $T$, denoted $\operatorname{rect}(T)$, is the straight shape tableau formed by shuffling $T$ with any straight shape tableau $S$. It is well known (often called the "fundamental theorem of jeu de taquin") that the result does not depend on $S$.

\subsection{Evacuation-shuffling and $\omega$}

Note that a straight-shape or rotated straight-shape has only one Littlewood-Richardson tableau, so an element of $\operatorname{LR}(\alpha, \square, \beta, \gamma)$ is essentially a pair $(\bigotimes, T)$, with $T$ a Littlewood-Richardson tableau of content $\beta$, and $\bigotimes$ an inner co-corner of $T$, such that the shape of $\bowtie \sqcup T$ is $\gamma^{c} / \alpha$. Computing esh( $(,, T)$ consists of the following steps: [9]

- Rectification. Treat the $囚$ as having value 0 and being part of a semistandard tableau $\widetilde{T}=\bigotimes \sqcup T$. Rectify, i.e. shuffle $(S, \widetilde{T})$ to $\left(\widetilde{T}^{\prime}, S^{\prime}\right)$, where $S$ is an arbitrary straight-shape tableau.

- Shuffling, or Promotion. (See [18] for the definition of promotion.) Delete the 0 of $\widetilde{T}^{\prime}$ and rectify the remaining portion of $\widetilde{T}^{\prime}$. Label the resulting empty outer corner with $\ell(\beta)+1$.

- Un-rectification. Un-rectify, i.e. shuffle once more with $S^{\prime}$. Replace the entry $\ell(\beta)+1$ by $\bigotimes$.

Note that the promotion step is equivalent to shuffling the $\otimes$ past the rest of the rectified tableau. Thus, evacuation-shuffling corresponds to conjugating the ordinary jeu de taquin shuffle (on skew tableaux) by rectifying the tableau. This procedure outputs an element $\left(T^{\prime}, \otimes\right) \in \operatorname{LR}(\alpha, \beta, \square, \gamma)$. Finally, the monodromy operator $\omega=$ sh $\circ$ esh is the commutator of rectification and shuffling.

\section{Local evacuation-shuffling}

We will now define local evacuation-shuffling,

$$
\text { local-esh : } \operatorname{LR}(\alpha, \square, \beta, \gamma) \rightarrow \operatorname{LR}(\alpha, \beta, \square, \gamma),
$$

a local algorithm for computing esh. The base case of our algorithm is the Pieri case, where $\beta$ is a one-row partition. In this case, esh was computed in Theorem 5.10 of [9], and we recall it here.

Theorem 3.1 (Pieri case) Let $\beta$ be a one-row partition. Then $\operatorname{esh}(\bowtie, T)$ exchanges $₫$ with the nearest $1 \in T$ prior to it in reading order, if possible. If there is no such 1 , esh instead exchanges $\square$ with the last 1 in reading order ( $a$ special jump).

We give two examples, illustrating the possible actions of esh and the more familiar sh.

1. If the skew shape contains a (necessarily unique) vertical domino:

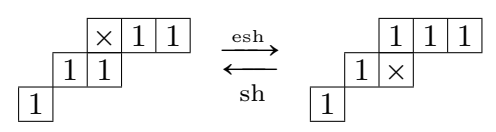

2. If it does not, the action of esh $\circ$ sh cycles the $\bigotimes$ through the rows of $\gamma^{c} / \alpha$ :

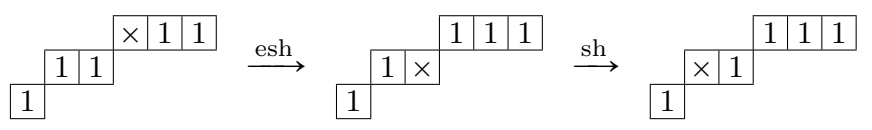




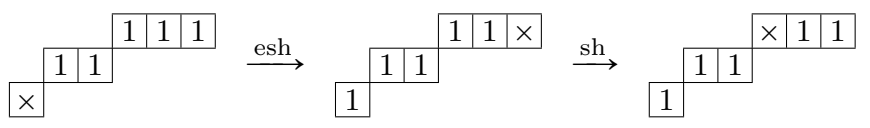

We refer the reader to [5] or [9] for two different proofs of this result.

\subsection{The algorithm}

We now state our new algorithm for evacuation-shuffling a box past an arbitrary ballot skew tableau.

Definition 3.2 Let $(\bowtie, T) \in \operatorname{LR}(\alpha, \square, \beta, \gamma)$. We define the local evacuation-shuffle, local-esh $(\bowtie, T)$, by the following algorithm, starting at $i=1$.

- Phase 1. If the $\square$ does not precede all of the i's in reading order, switch $\square$ with the nearest $i$ prior to it in reading order. Then increment $i$ by 1 and repeat Phase 1.

If, instead, the $₫$ precedes all of the i's in reading order, go to Phase 2.

- Phase 2. If the suffix from $₫$ is not tied for $(i, i+1)$, switch $\bowtie$ with the nearest $i$ after it in reading order. Repeat this process until the suffix becomes tied for $(i, i+1)$. Then increment $i$ by 1 and repeat Phase 2 until $i=\ell(\beta)+1$.

We say that $s$ is the transition step if the algorithm switches to Phase 2 while $i=s$. If the algorithm remains in Phase 1 throughout, we say the transition step is $s=\ell(\beta)+1$.

Remark 3.3 Phase 1 is identical to the Pieri case unless the Pieri case calls for a special jump.

In Phase 1, $₫$ moves down and to the left; in Phase 2, $₫$ instead moves to the right and up. (See Figure 2) We refer to the squares occupied by the box during the algorithm as the evacu-shuffle path.

Note that in Phase 2, it is not obvious that we can find an $i$ after the $\square$ in reading order. However, in [5] we show the following lemma, which states that the tableau essentially remains semistandard and ballot at each step of the algorithm. Consequently, the topmost $i$ is such a square.

Lemma 3.4 Let $T_{i}$ be the tableau before the $i$-th step. Then, omitting the $₫$, the rows (columns) of $T$ are weakly (strictly) increasing and the reading word of $T$ is ballot.

Example 3.5 The diagram below demonstrates the local-esh algorithm.

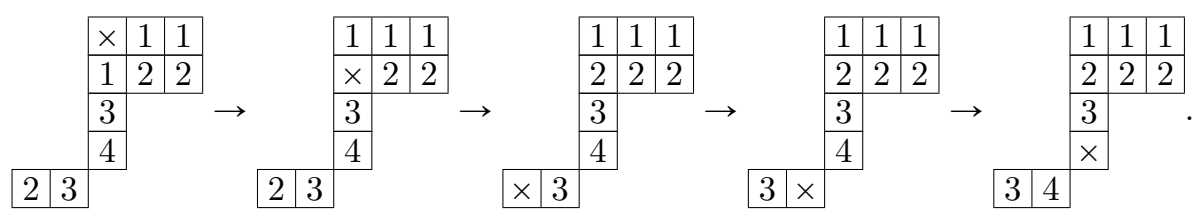




\subsection{Proof of Theorem 1.3}

In this section we outline the proof of the following:

Theorem 3.6 Local evacuation-shuffling agrees with evacuation-shuffing, that is, for any $(囚, T)$,

$$
\text { local-esh }(\bowtie, T)=\operatorname{esh}(\bowtie, T) .
$$

The main idea is as follows. In computing esh, when we first rectify $(囚, T)$, we obtain a tableau $R$ of the form shown in Figure 3 In particular, the $\otimes$ is in the corner and the total shape of $₫ \sqcup R$ is formed by adding an outer corner square to $\beta$ in some row $s$.

When shuffling the $\otimes$ past $R$, the $\otimes$ follows a path directly down to row $s$ and then directly over to the end of row $s$, as shown. It turns out that this corresponds to a more refined process in which we shuffle the $\bowtie$ past rows $1,2, \ldots, s-1$, then shuffle it past the $\beta_{s}$ vertical strips formed by greedily taking vertical strips from the right of the remaining tableau. We call this decomposition into horizontal and vertical strips the $s$-decomposition, and we can similarly form the $s$-decomposition of the (unrectified, skew) tableau $T$ into horizontal and vertical strips.

Each step of Phase 1 of local-esh corresponds to a single move of the $₫$ past a horizontal strip in the $s$ decomposition of $\beta$; the transition step then turns out to be $s$. Using the antidiagonal symmetry suggested by Figure 2, we show that the movements of the $\otimes$ during Phase 2 correspond similarly to shuffles past each of the $s$-decomposition's vertical strips.

The complete proof given in [5] uses the theory of dual equivalence classes of tableaux (developed in [6]), which are in bijection with Littlewood-Richardson tableaux. The theory of dual equivalence allows us to use outwards rather than inwards rectification to compute esh, which leads to the observed antidiagonal symmetry.
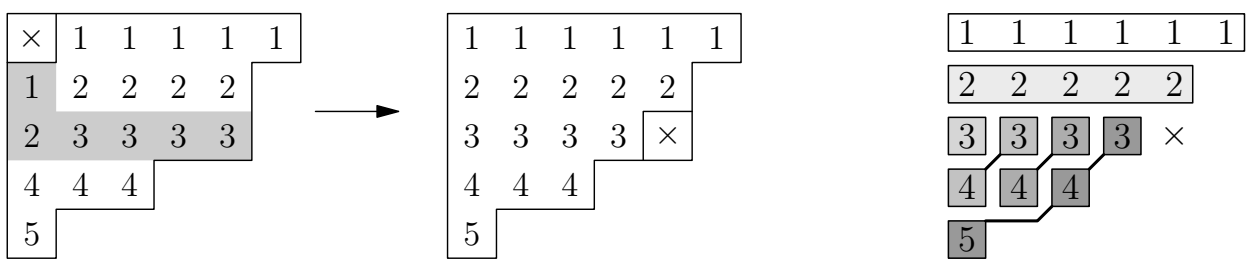

Figure 3: An example of a rectified tableau $R$ with transition step $s=3$. The promotion path of the box is down to row $s$ and then directly right. The corresponding $s$-decomposition is shown at right.

\section{Connections to K-theory}

\subsection{Generating K-theoretic tableaux}

We recall the results we need on K-theory. The structure sheaves $\mathcal{O}_{\lambda}$ of Schubert varieties in $\operatorname{Gr}\left(k, \mathbb{C}^{n}\right)$ form an additive basis for the $\mathrm{K}$-theory ring $K\left(G r\left(k, \mathbb{C}^{n}\right)\right)$, with a product formula

$$
\left[\mathcal{O}_{\alpha}\right] \cdot\left[\mathcal{O}_{\beta}\right]=\sum_{\left|\gamma^{c}\right| \geqslant|\alpha|+|\beta|}(-1)^{\left|\gamma^{c}\right|-|\alpha|-|\beta|} k_{\alpha \beta}^{\gamma^{c}}\left[\mathcal{O}_{\gamma^{c}}\right]
$$


for certain nonnegative integer coefficients $k_{\alpha \beta}^{\gamma^{c}}$. In [12], Pechenik and Yong introduced genomic tableaux to enumerate $k_{\alpha \beta}^{\gamma^{c}}$, a 'ballot semistandard' analog of Thomas and Yong's earlier theory [15] of increasing tableaux. We state an equivalent characterization of genomic tableaux when $\left|\gamma^{c}\right|-|\alpha|-|\beta|=1$.

Lemma/Definition 4.1 Let $T$ be an (ordinary) semistandard tableau of shape $\gamma^{c} / \alpha$ and content equal to $\beta$ except for a single extra $i$. Let $\left\{\bigotimes_{1}, \bigotimes_{2}\right\}$ be a pair of squares of $T$. The data $\left(T,\left\{\bigotimes_{1}, \bigotimes_{2}\right\}\right)$ corresponds to a ballot genomic tableau if

(i) The squares are non-adjacent and contain $i$,

(ii) There are no i's between $\bigotimes_{1}$ and $\bigotimes_{2}$ in the reading word of $T$,

(iii) For $k=1,2$, the word obtained by deleting $\bigotimes_{k}$ from the reading word of $T$ is ballot.

We say that the K-theoretic content is $\beta$. We write $K\left(\gamma^{c} / \alpha ; \beta\right)$ for the set of ballot genomic tableaux of shape $\gamma^{c} / \alpha$ and $K$-theoretic content $\beta$, and $K\left(\gamma^{c} / \alpha ; \beta\right)(i)$ for the tableaux whose extra entry is $i$.

Theorem 4.2 ([12]) We have $k_{\alpha \beta}^{\gamma^{c}}=\left|K\left(\gamma^{c} / \alpha ; \beta\right)\right|$.

We now describe how local-esh generates genomic tableaux.

Theorem 4.3 Let $\bigotimes_{1}, \bigotimes_{2}$ be two successive non-adjacent squares in the evacu-shuffle path of $(\bowtie, T)$ in which the $\square$ switches with an $i$. Let $T_{i}$ be the tableau before this step in the path, with the $\otimes$ replaced by $i$. Then the data $\left(T_{i},\left\{\bigotimes_{1}, \bigotimes_{2}\right\}\right)$ corresponds to a ballot genomic tableau $T_{K}$, as in Lemma 4.1 .

Moreover, as $T$ ranges over $\operatorname{LR}(\alpha, \square, \beta, \gamma)$, every tableau $T_{K} \in K\left(\gamma^{c} / \alpha ; \beta\right)(i)$ arises exactly once this way in Phase 1 and once more in Phase 2. This gives two bijections:

$$
\varphi_{1}, \varphi_{2}:\{\text { non-adjacent } \bowtie \text { movements past an } i\} \leftrightarrow K\left(\gamma^{c} / \alpha ; \beta\right)(i) \text {. }
$$

In Example 3.5, the second step generates a genomic tableau for $\varphi_{1}$ and the fourth generates one for $\varphi_{2}$.

\subsection{The sign and reflection length of $\omega$}

We now compute the sign of $\omega=\operatorname{sh} \circ$ esh, as a permutation of $\operatorname{LR}(\alpha, \square, \beta, \gamma)$, and the bound (3) on its reflection length. We show:

Theorem 4.4 We have rlength $(\omega) \leqslant\left|K\left(\gamma^{c} / \alpha ; \beta\right)\right|$ and $\operatorname{sgn}(\omega) \equiv\left|K\left(\gamma^{c} / \alpha ; \beta\right)\right|(\bmod 2)$.

Lemma 4.5 Let $X_{i}$ and $X_{i}^{\prime}$, respectively, be the set of all tableaux arising in local-esh and sh, respectively, when the $囚$ is between the $(i-1)$-st and $i$-th horizontal strips. Then $X_{i}=X_{i}^{\prime}$.

Proof: Both sets consist of 'punctured' semistandard tableaux of content $\beta$ and shape $\gamma^{c} / \alpha$, with ballot reading word, and where the $₫$ is between the $(i-1)$-st and $i$-th horizontal strips. (See Lemma 3.4.)

We have $X_{1}=\operatorname{LR}(\alpha, \square, \beta, \gamma)$ and we write $X_{t+1}=\operatorname{LR}(\alpha, \beta, \square, \gamma)$, where $t=\ell(\beta)$. For $1 \leqslant i \leqslant t$, we let $\ell_{i}: X_{i} \rightarrow X_{i+1}$ be the composition of the steps of local-esh that switch the $\otimes$ with $i$ 's. Let $s_{i}: X_{i+1} \rightarrow X_{i}$ be the jeu de taquin shuffle. We have the diagram

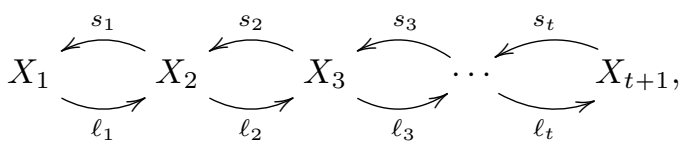


By definition, $\omega=\operatorname{sh} \circ$ local-esh $=s_{1} \circ \cdots \circ s_{t} \circ \ell_{t} \circ \cdots \circ \ell_{1}$. It follows directly that

$$
\begin{aligned}
\operatorname{sgn}(\omega) & \equiv \sum_{i=1}^{t} \operatorname{sgn}\left(s_{i} \circ \ell_{i}\right) \quad(\bmod 2), \\
\operatorname{rlength}(\omega) & \leqslant \sum_{i=1}^{t} \operatorname{rlength}\left(s_{i} \circ \ell_{i}\right) .
\end{aligned}
$$

The operators $\omega_{i}$ of Theorem 1.6 are the compositions $\omega_{i}=s_{1} \cdots s_{i-1}\left(s_{i} \ell_{i}\right) s_{i-1} \cdots s_{1}$. We complete Theorem 4.4 by describing the orbits of $s_{i} \circ \ell_{i}$, a computation interesting in its own right:

Theorem 4.6 Let orb $_{i}$ be the set of orbits of $s_{i} \circ \ell_{i}$. Then:

$$
\operatorname{rlength}\left(s_{i} \circ \ell_{i}\right)=\sum_{\mathcal{O} \in \operatorname{orb}_{i}}(|\mathcal{O}|-1)=\left|K\left(\gamma^{c} / \alpha ; \beta\right)(i)\right| .
$$

Proof sketch: We use the bijection $\varphi_{1}$ of Theorem 4.3 to generate genomic tableaux. Let $T \in X_{i}$.

There are two cases. First, if $\ell_{i}$ does not involve a non-adjacent move, it is easy to check that $\ell_{i}(T)=$ $s_{i}^{-1}(T)$, so $T$ is a fixed point and does not contribute to either side of the sum.

Otherwise, the orbit resembles the Pieri case: all steps but one move the $\square$ down one row, giving a single genomic tableau; the last step begins in Phase 2 and resets the location of the $凶$, giving no genomic tableaux. (Unlike the Pieri case, the $\square$ moves downwards only until the $(i-1, i)$ suffix becomes tied, and 'jumps' only far enough upwards to make the $(i, i+1)$ suffix tied.) Thus each $\mathcal{O} \in \operatorname{orb}_{i}$ generates exactly $|\mathcal{O}|-1$ genomic tableaux. Every tableau of $K\left(\gamma^{c} / \alpha ; \beta\right)(i)$ arises once in Phase 1, so we are done.

\subsection{Fixed points of $\omega$}

We also characterize the fixed points of $\omega$ :

Proposition 4.7 The fixed points of $\omega$ are the pairs $(\bigotimes, T)$ satisfying the (equivalent) conditions:

(i) In the computation of local-esh $(\bowtie, T)$, neither bijection $\varphi_{1}, \varphi_{2}$ generates a genomic tableau.

(ii) The evacu-shuffle path of the $\otimes$ is connected.

Corollary 4.8 Suppose $\omega$ acts on $\operatorname{LR}(\alpha, \square, \beta, \gamma)$ as the identity. Then $K\left(\gamma^{c} / \alpha ; \beta\right)=\varnothing$; it follows that the curve $S(\alpha, \beta, \gamma)$ is (over $\mathbb{C}$ ) a disjoint union of $\mathbb{P}^{1}$ 's, and the map $S \rightarrow \mathbb{P}^{1}$ of Theorem 1.2 is locally an isomorphism.

We note that in general, a morphism of real algebraic curves $C \rightarrow D$, inducing a covering map on real points, may have trivial real monodromy but be algebraically nontrivial (i.e., not be a local isomorphism). Corollary 4.8 shows that this cannot occur for Schubert curves.

\section{Geometric constructions}

It is considerably easier to analyze the orbit structure of $\omega$, and, therefore, the geometric structure of the Schubert curve, using local-esh. As examples, we give two families of triples $(\alpha, \beta, \gamma)$ for which the Schubert curve $S(\alpha, \beta, \gamma)$ exhibits 'extremal' numerical and geometrical properties. See [5] for full proofs, which rely on the relative simplicity of local-esh. 
Example 5.1 (Schubert curves of high genus) Let $t \geqslant 2$ be a positive integer. Let

$$
\boxplus=(t+2)^{t+1} ; \quad \alpha=\gamma=(t, t-1, t-2, \ldots, 2,1) ; \quad \beta=\left(t+1,2,1^{t-2}\right)
$$

so $\gamma^{c} / \alpha$ is a staircase-ribbon. Then $\omega$ has only one orbit on $\operatorname{LR}(\alpha, \square, \beta, \gamma)$. Hence, the Schubert curve $S_{t} \subset G\left(t+1, \mathbb{C}^{2 t+3}\right)$ is integral; moreover, its arithmetic genus is $g\left(S_{t}\right)=(t-1)(t-2)$.

In [9], the second author asked if Schubert curves are always smooth. K-theory does not in general detect singularities, but either possibility is interesting: that $S_{t}$ gives examples of singular Schubert curves for $t \gg 0$, or that it gives smooth Schubert curves of arbitrarily high (geometric) genus.

Example 5.2 (Schubert curves with many connected components) Let $t \geqslant 2$ be a positive integer. Let

$$
\boxplus=(t+1)^{t+1} ; \quad \alpha=(t, t-1, \ldots, 2) ; \quad \beta=(t, 1,1) ; \quad \gamma=(t+1, t, \ldots, 3,2,2)
$$

Then $\omega$ acts as the identity on $\operatorname{LR}(\alpha, \square, \beta, \gamma)$, which has $t-1$ elements. Consequently, the Schubert curve $S_{t} \subset G\left(t+1, \mathbb{C}^{2 t+2}\right)$ is a disjoint union of $t-1$ copies of $\mathbb{P}^{1}$.

\section{Conjectures}

Numerical evidence suggests that the inequality (3) in fact 'holds orbit-by-orbit' for $\omega$ :

Conjecture 6.1 Using the bijections $\varphi_{1}, \varphi_{2}$ of Theorem 4.3 each orbit $\mathcal{O}$ of $\omega$ generates at least $|\mathcal{O}|-1$ genomic tableaux.

Conjecture 6.1 implies the inequality (3), by summing over the orbits of $\omega$. We have verified it for orbits of size at most 3, and (computationally) for $n \leqslant 10$ (for all choices of $k, \alpha, \beta$, and $\gamma$ ). We have also established the conjecture in certain special cases:

Theorem 6.2 Conjecture 6.1 holds for $\varphi_{1}$ if $\beta$ has two rows, and for $\varphi_{2}$ if $\beta$ has two columns.

Finally, although we have only defined local evacuation-shuffling for Littlewood-Richardson tableaux, the evacuation-shuffle esh is defined on all tableaux $(\bowtie, T)$ as the conjugation of shuffling by rectification. Our results do yield local algorithms for certain other Knuth classes of tableaux via straightforward alterations to local-esh. It would be interesting to understand the actions of esh and $\omega$ on semistandard tableaux in general, and to extend the connection to K-theoretic Schubert calculus. To be precise:

Conjecture 6.3 Let $T$ be any (semi)standard skew tableau and $₫$ an inner co-corner of $T$. There exists a local algorithm for computing esh $(\bigotimes, T)$, which does not require rectifying the tableau, such that:

(i) Each step consists of exchanging the $₫$ with an entry of $T$, of weakly increasing value.

(ii) The Knuth equivalence class of the word of $T$ (omitting $区$ ) is preserved throughout the algorithm.

(iii) The algorithm specializes to jeu de taquin (if $T$ is of straight shape) and local-esh (if $T$ is ballot).

Each step should correspond (by conjugating with rectification) to a jeu de taquin slide of $₫$ through the rectification $\operatorname{rect}(\bowtie, T)$.

For a straight-shape tableau $T$ that is not highest-weight, it would be interesting to find an analog of the $s$-decomposition to describe the path of the $凶$, and to use it to give a local algorithm on any skew tableau $T^{\prime}$ whose rectification is $T$. Finally, we ask how to compute $\operatorname{esh}(S, T)$ locally, where both $S$ and $T$ may have more than one box. 


\section{Acknowledgements}

We thank David Speyer, Oliver Pechenik, and Mark Haiman for many helpful conversations.

\section{References}

[1] A. D. Berenstein, A. N. Kirillov, Domino tableaux, Schützenberger involution and action of the symmetric group, Discrete Math., 225(1-3):5-24, 2000.

[2] M. Chan, A. López Martın, N Pflueger, M. Teixidor i Bigas. Genera of Brill-Noether curves and staircase paths in Young tableaux. Preprint, arXiv:1506.00516, 2015.

[3] D. Eisenbud and J. Harris, Limit linear series: Basic theory, Invent. Math., 85(2):337-371, 1986.

[4] W. Fulton, Young Tableaux, London Math. Soc. Stud. Texts 35, Cambridge University Press, 1997.

[5] M. Gillespie, J. Levinson, Monodromy and $K$-theory of Schubert curves via generalized jeu de taquin, in preparation.

[6] M. Haiman, Dual equivalence with applications, including a conjecture of Proctor, Discrete Math. 92 (1992), 79-113.

[7] A. Henriques, J. Kamnitzer, Crystals and coboundary categories, Duke Math. J., 132 (2):191-216, 2006.

[8] A. N. Kirillov, A. D. Berenstein, Groups generated by involutions, Gelfand-Tsetlin patterns, and combinatorics of Young tableaux, Algebra i Analiz, 7(1):92-152, 1995.

[9] J. Levinson, One-dimensional Schubert problems with respect to osculating flags, Preprint, arXiv:1504.06542.

[10] E. Mukhin, V. Tarasov, and A. Varchenko, Schubert calculus and representations of the general linear group, J. Amer. Math. Soc., 22(4):909-940, 2009.

[11] B. Osserman, A limit linear series moduli scheme. Ann. Inst. Four., 56(4):1165-1205, 2006.

[12] O. Pechenik, A. Yong, Equivariant $K$-theory of Grassmannians, Preprint, arXiv:1506.01992.

[13] K. Purbhoo, Jeu de taquin and a monodromy problem for Wronskians of polynomials, Adv. Math., 224(3):827-862, 2010.

[14] K. Purbhoo, Wronskians, cyclic group actions, and ribbon tableaux, Trans. Amer. Math. Soc., 365 (2013), 1977-2030.

[15] H. Thomas, A. Yong, A jeu de taquin theory for increasing tableaux, with applications to K-theoretic Schubert calculus, Algebra Number Theory 3 (2009), no. 2, 121-148.

[16] F. Sottile, Frontiers of reality in Schubert calculus, Bull. Amer. Math. Soc., 47(1):31-71, 2010.

[17] D. Speyer, Schubert problems with respect to osculating flags of stable rational curves, Algebraic Geometry, 1:14-45, 2014.

[18] R. Stanley, Enumerative Combinatorics, Vol. 2, Cambridge University Press (1999). 